\title{
Sleep-time Sizing and Scheduling in Green Passive Optical Networks
}

\author{
Amr Elrasad, Mohammad G. Khafagy, Basem Shihada \\ King Abdullah University of Science and Technology (KAUST), Thuwal, Saudi Arabia \\ Email: \{amr.rasad, mohammad.khafagy, basem.shihada\}@kaust.edu.sa
}

\begin{abstract}
Next-generation passive optical network (PON) has been widely considered as a cost-effective broadband access technology. With the ever-increasing power saving concern, energy efficiency has been an important issue for its operations. In this paper, we present a novel sleep time sizing and scheduling framework that satisfies power efficient bandwidth allocation in PONs. We consider the downstream links from an optical line terminal (OLT) to an optical network unit (ONU). The ONU has two classes of traffic, control and data. Control traffic are delay intolerant with higher priority than the data traffic. Closed form model for average ONU sleeping time and end-to-end data traffic delay are presented and evaluated. Our framework decouples the dependency between ONU sleeping time and the QoS of the traffic.
\end{abstract}

\section{INTRODUCTION}

Energy consumption is emerging as a major challenge in today's information and communication technologies. The expansion of data communication usage in different applications has resulted in a tremendous increase in energy consumption worldwide. According to recent statistics, the energy cost to operate wireless devices accounts for half of the operating expenses for any wireless deployment. Currently $3 \%$ of the world-wide energy is consumed by the information and communication technology infrastructures that cause about $2 \%$ of the world-wide $\mathrm{CO}_{2}$ emissions. This figure is comparable to the worldwide $\mathrm{CO}_{2}$ emissions by airplanes or $25 \%$ of the world-wide $\mathrm{CO}_{2}$ emissions caused by cars. Therefore, making communication devices energy efficient will not only benefit the environment, but also increase the profitability of business for communication companies.

In order to fulfill the bandwidth-thirsty demands and achieve the best operational efficiency, we are experiencing fast development of fiber-wireless (FiWi) access technologies [1][5], which are envisioned to serve as the enabling technologies for the next-generation metropolitan-area networks. Such infrastructure aims to take the advantage of an integrated design of the optical and broadband wireless access systems in both phases of network planning/dimensioning and real-time operations.

Passive optical networks (PONs) have been introduced as the most promising approach for solving the first mile problem in broadband access networks [6]. As shown in Fig. 1, the structure of PON consists of an optical line Terminal (OLT), located at a cental office (CO) that is connected to a set of optical network units (ONUs) located at the end-user locations [8]. In the past decade, PON has been a subject of extensive

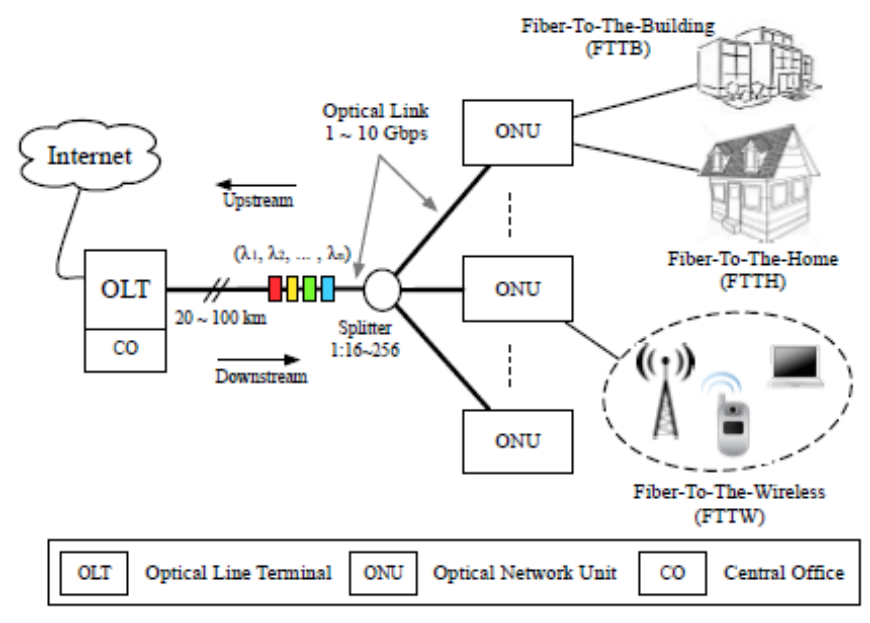

Fig. 1. PON System

study concerning quality of service (QoS), dynamic bandwidth allocation (DBA), and recently energy saving mechanisms. In PON, energy saving can be achieved by putting ONU into sleep mode while watching the network QoS condition. Most of the DBA studies assume that all ONUs can sleep except the one transmitting data to the OLT [7]- [11]. The fundamental assumption in all of these studies is that the OLT can buffer the downstream traffic of the sleeping ONU until it returns back to the operational mode. It is important to obtain the optimal sleeping time while considering certain traffic QoS requirements. In [12], the authors developed a new MAC-Layer protocol that uses fixed bandwidth allocation scheme under low load operation. The OLT goes to the energy saving mode if it finds that the throughput is below certain threshold. In sleeping mode, each ONU wakes-up periodically in predefined periods to send and receive upstream and downstream traffic, respectively. The authors in [13] tackled the energy saving problem in Gigabit-PON (GPON) using the adaptive link rate (ALR) approach. They have used a simple mechanism to enable the ONU to adaptively change the optical link rate based on the traffic load to increase the sleep mode period. However, the proposed scheme did not consider any specific traffic QoS requirements. Two energy saving downstream MAC-layer schemes are proposed in [14]. In the first scheme, which is called downstream centric (DCS), the downstream traffic for certain ONU is buffered until an 
upstream time slot is assigned to it. In the second scheme, which is called the upstream centric (UCS), the ONU switches to active mode whenever there is an upstream or downstream transmission slots scheduled. Although UCS achieves better energy saving, it causes higher delay when downstream and upstream time slots are not efficiently scheduled. The work in [15] developed a downstream traffic scheduling protocol with the limited service discipline under the UCS-based green bandwidth allocation framework. The developed scheme investigates the maximum sleep time of each ONU and maximum possible energy saving.

In our work, we present a simplified, yet effective, novel PON scheme that supports energy saving. Our analytical and simulation results show that our scheme saves energy without introducing significant delay to the network traffic. The main advantage of our scheme is that the OLT allocates the bandwidth for each ONU considering the downstream buffer size. In other words, the ONU remains in the sleep mode until its downstream buffer reaches a certain threshold. The OLT uses a separate control traffic plane to wake-up the ONU.

The rest of the paper is organized as follows. The proposed architecture is described in Section II. In Section III, we present the adopted analytic model to estimate the suitable buffer threshold for a given QoS delay constraint. Numerical results and simulations are presented in Section IV. Finally, conclusions are drawn in Section V.

\section{SLEEP SizING FrAmEWORK}

In this section, we provide an overview of our proposed sleep sizing scheme. This includes the signalling mechanism between the OLT and ONUs.

\section{A. Overview}

Our scheme uses conventional PON tree topology and assumes that the OLT and ONU signaling messages are exchanged over a separate control plane. Also, we assume that the downstream traffic is larger than the upstream one, hence the ONU wake-up by OLT according to the downstream queue size threshold. Upon reaching certain threshold, OLT uses the control plane to wake-ups the ONU for serving both downstream and upstream traffic. Two classes of packets are received by the ONUs called control and data packets. Control packets are assumed to be delay intolerant, while data packets can tolerate certain average delay constraint $\mathbb{D}_{Q o S}$. Thus, control packets are assumed to be of higher priority than data packets. Furthermore, the queuing discipline within each class is assumed to be FIFO, while the service prioritization between classes is based on non-preemptive service. Moreover, the ONU is allowed to sleep for energy saving purposes. Initially, ONU remains in the sleep mode until either a control packet arrives or data queue size reaches certain threshold $Q_{t h}$, which depends on the average delay constraint $\mathbb{D}_{Q o S}$ in addition to the arrival and service parameters. This is known to be an $N$-threshold policy queuing system [16]. Later in this section, we obtained a closed form solution for the average sleeping time duration and the percentage of time ONU spends in the sleeping mode to obtain the energy saving gain. Also, we obtained a numerical solution for the data queue threshold $Q_{t h}$ computed in terms of arrival rate, service rate, and average delay constraint.

\section{B. System Parameters}

1) Arrival and service processes: Control and data arrival processes are assumed to be independent poisson processes of rates, $\lambda_{c}$ and $\lambda_{d}$, respectively. Their service is assumed to be generally distributed. The mean transmission time for control and data packets is given by $\overline{X_{c}}$ and $\overline{X_{d}}$ respectively. Their corresponding reciprocals are given respectively by $\mu_{c}$ and $\mu_{d}$ denoting the service rates. The second moments of the service time for control and data packets are given by $\overline{X_{c}^{2}}$ and $\overline{X_{d}^{2}}$, respectively.

2) Data packets delay constraint: The framework assumes that data packets can tolerate an average delay constraint $\mathbb{D}_{Q o S}$. This allows the ONU to sleep as long as there is no control packets to serve and the data queue size is below the threshold.

\section{Notation}

Each ONU sleeping cycle is composed of two successive periods, where it begins by a sleeping period for random duration $T_{s}$ followed by an active period for random duration $T_{a}$. The corresponding averages are denoted by $\overline{T_{s}}$ and $\overline{T_{a}}$, respectively. Their probability distributions are denoted by $f_{s}$ and $f_{a}$, while $F_{s}$ and $F_{a}$ are the cumulative distributions. When needed, $\overline{F_{i}}$ would denote the complementary cumulative density function associated with $F_{i}$. Table I summarizes the notation used in our analytical model.

TABLE I

NOTATION

\begin{tabular}{l|l}
\hline$\lambda_{i}$ & Poisson arrival rate of class $i \in\{c, d\}$ \\
$\lambda_{t o t}$ & Sum of control and data arrival rates $=\lambda_{c}+\lambda_{d}$ \\
$X_{i}$ & Random variable for service time of class $i \in\{c, d\}$ \\
$\overline{X_{i}}$ & Mean service time of class $i \in\{c, d\}$ \\
$\overline{X_{i}^{2}}$ & Second moment of service time of class $i \in\{c, d\}$ \\
$\overline{W_{d}}$ & Average waiting time of data packets in the queue \\
$\overline{D_{d}}$ & Average arrival-to-departure data packet delay \\
$\mu_{i}$ & Service rate of class $i=\frac{1}{X_{i}}$ \\
$\rho_{i}$ & Utilization of class $i=\frac{\lambda_{i}}{\mu_{i}}$ \\
$\mathbb{D}_{Q o S}$ & Average delay constraint for data packets \\
$Q_{i}(t)$ & Queue length of class $i$ at time $t$ \\
$Q_{t h}$ & Data queue threshold depending on the delay con- \\
& straint \\
$T_{s}$ & ONU sleep period duration random variable \\
$T_{a}$ & ONU active period duration random variable \\
$\overline{T_{s}}$ & Average ONU sleeping time \\
$f_{s}$ & Probability distribution of sleeping time \\
$f_{a}$ & Probability distribution of active time \\
$F_{s}$ & Cumulative distribution of sleeping time \\
$F_{a}$ & Cumulative distribution of active time \\
$F_{a}$ & Complementary cumulative distribution of active time \\
$S(t)$ & state of ONU at time $t$ (0: sleep, 1: active) \\
\hline
\end{tabular}




\section{ANALYTiCAL MODEL}

\section{A. Average sleeping time}

In this section, we derive an expression for the probability distribution of the ONU sleeping time. Once the distribution is derived, we accordingly present a closed form expression for the average ONU sleeping time as a function of the data queue threshold and the arrival rates of data and control packets. The probability distribution is derived in three phases based on the independence of the traffic arrival processes of control and data packets. First, we consider that the system is serving data packets only. Second, we consider that the system is serving control packets only. Finally, we combine the two processes to get an expression for the distribution of ONU sleeping time with both arrivals allowed. For each of the above system phases, we present the PDF and CDF of the sleeping time. Accordingly, we average the obtained distribution to get the ONU average sleeping time $\overline{T_{s}}$.

1) System with data packet arrivals: In the absence of control packet arrivals, the ONU remains sleeping until the arrival of certain number of data packets equal to $Q_{t h}$. Since the arrival process is assumed to be Poisson distributed with rate $\lambda_{d}$, the interarrival time distribution is exponentially distributed with mean $\frac{1}{\lambda_{d}}$. In this case, the time required to reach $Q_{t h}$ packets in the queue for the ONU to wake-up is equal to the sum of $Q_{t h}$ i.i.d exponential random variable with mean $\frac{1}{\lambda_{d}}$ (i.e., average interarrival time). Thus, the sleeping time has an Erlang distribution that is given by,

$$
f_{(s, d)}\left(t ; Q_{t h}, \lambda_{d}\right)=\frac{\lambda_{d}^{Q_{t h}} t^{Q_{t h}-1} e^{-\lambda_{d} t}}{\left(Q_{t h}-1\right) !}
$$

while the corresponding cumulative density function is given by,

$$
F_{(s, d)}\left(t ; Q_{t h}, \lambda_{d}\right)=1-\sum_{n=0}^{Q_{t h}-1} \frac{e^{-\lambda_{d} t}\left(\lambda_{d} t\right)^{n}}{n !}
$$

2) System with control packet arrivals: In the absence of data packet arrivals, the allowable time for an ONU to sleep is the interarrival time of control packets which is exponentially distributed with mean $\frac{1}{\lambda_{c}}$, assuming the service time is very small compared to the interarrival time. ${ }^{1}$ Thus, the probability density function is given by.

$$
f_{(s, c)}\left(t ; \lambda_{c}\right)=\lambda_{c} e^{-\lambda_{c} t}
$$

while the corresponding cumulative density function is given by.

$$
F_{(s, c)}\left(t ; \lambda_{c}\right)=1-e^{-\lambda_{c} t}
$$

3) System with both control and data packet arrivals: Since the ONU does not go to sleep mode except when there is no packets in the queue, we consider the start of the sleeping cycle as a reference point in the following analysis (i.e., $t=0$ at the beginning of the sleep cycle). Thus, $Q_{c}(0)=Q_{d}(0)=0$.

\footnotetext{
${ }^{1}$ The arrival rate of control packets is expected to be small leading to relatively long interrival time
}

Sleeping time can be equal to $T_{s}$ in two cases only. First case occurs when there is no control arrivals $\forall t \in\left[0, T_{s}\right]$ while the $Q_{t h}$ data packet arrives at $t=T_{s}$. The second case occurs when the data queue size is less than $Q_{t h} \forall t \in\left[0, T_{s}\right]$ while a control packet arrives at $t=T_{s}$. This can be expressed using the previously derived PDF and CDF expressions. Using the independence between the two processes in which the joint distributions become the product of the marginal ones is shown below,

$$
\begin{aligned}
f_{s}\left(T_{s}\right)= & f_{(s, d)}\left(T_{s}\right) \overline{F_{(s, c)}}\left(T_{s}\right)+f_{(s, c)}\left(T_{s}\right) \overline{F_{(s, d)}}\left(T_{s}\right) \\
= & \frac{\lambda_{d}^{Q_{t h}} T_{s}^{Q_{t h}-1} e^{-\lambda_{d} T_{s}}}{\left(Q_{t h}-1\right) !} e^{-\lambda_{c} T_{s}} \\
& +\lambda_{c} e^{-\lambda_{c} T_{s}} \sum_{n=0}^{Q_{t h}-1} \frac{e^{-\lambda_{d} T_{s}}\left(\lambda_{d} T_{s}\right)^{n}}{n !} \\
= & \lambda_{d}^{Q_{t h}} \frac{T_{s}^{Q_{t h}-1} e^{-\lambda_{t o t} T_{s}}}{\left(Q_{t h}-1\right) !} \\
& +\sum_{n=0}^{Q_{t h}-1} \lambda_{c} \lambda_{d}^{n} \frac{T_{s}^{n} e^{-\lambda_{t o t} T_{s}}}{n !} \\
\overline{T_{s}}= & \int_{0}^{\infty} t f_{s}(t) d t \\
= & \frac{\lambda_{d}^{Q_{t h}}}{\left(Q_{t h}-1\right) !} \int_{0}^{\infty} t^{Q_{t h}} e^{-\lambda_{t o t} t} d t \\
& +\sum_{n=0}^{Q_{t h}-1} \frac{\lambda_{c} \lambda_{d}^{n}}{n !} \int_{0}^{\infty} t^{n+1} e^{-\lambda_{t o t} t} d t
\end{aligned}
$$

By changing the variables and using Gamma function, we obtain the above integral to be equal to,

$$
\int_{0}^{\infty} t^{n+1} e^{-\lambda_{t o t} t} d t=\frac{(n+1) !}{\lambda_{t o t}^{n+2}}
$$

Thus, the average sleeping time is given by the closed form expression as follows,

$$
\begin{aligned}
\overline{T_{s}} & =Q_{t h} \frac{\lambda_{d}^{Q_{t h}}}{\lambda_{t o t}^{Q_{t h}+1}}+\sum_{n=0}^{Q_{t h}-1} \frac{(n+1) \lambda_{c} \lambda_{d}^{n}}{\lambda_{t o t}^{n+2}} \\
& =\frac{1-\left(\frac{\lambda_{d}}{\lambda_{c}+\lambda_{d}}\right)^{Q_{t h}}}{\lambda_{c}}
\end{aligned}
$$

From the above, we notice that the average sleeping time is a function of the control and data packet arrival rates and the allowable data queue threshold. However, we still need to find the average active time in order to know the percentage of time the ONU is allowed to sleep without degrading QoS.

We denote the average sleeping time by $\overline{T_{s}}$ and the average number of data and control arrivals within this period is given by $\lambda_{d} \overline{T_{s}}$ and $\lambda_{c} \overline{T_{s}}$. Within the active period $\overline{T_{a}}$, the expected number of data and control arrivals is equal to $\lambda_{c} \overline{T_{a}}$ and $\lambda_{c} \overline{T_{a}}$, respectively. Each data packet is served with an average service time $\overline{X_{d}}$, while the control packets have an average service time of $\overline{X_{c}}$. Hence, the ONU should remain active 
until the queue is empty. This can be expressed as follows,

$$
\begin{aligned}
\overline{T_{a}} & =\lambda_{c} \overline{T_{s} X_{c}}+\lambda_{d} \overline{T_{s} X_{d}}+\lambda_{c} \overline{T_{a} X_{c}}+\lambda_{d} \overline{T_{a} X_{d}} \\
& =\left(\rho_{c}+\rho_{d}\right) \overline{T_{s}}+\left(\rho_{c}+\rho_{d}\right) \overline{T_{a}}
\end{aligned}
$$

Thus,

$$
\overline{T_{a}}=\frac{\rho_{c}+\rho_{d}}{1-\rho_{c}-\rho_{d}} \overline{T_{s}}
$$

We write the equation (9) in the following alternative form,

$$
\frac{\overline{T_{a}}}{\overline{T_{a}}+\overline{T_{s}}}=\rho_{c}+\rho_{d}
$$

This form states that the ONU is only active for a fraction of time, which is equal to the introduced aggregate load. This fact is one of the main advantages of our framework since it shows that we successfully decoupled the sleeping time from the QoS constraints. In other words, the average sleeping period depends only on the traffic load. Using (5), we can evaluate the second moment of sleeping time as follows,

$$
\overline{T_{s}^{2}}=\frac{2\left[1-\left(Q_{t h}+1\right)\left(\frac{\lambda_{d}}{\lambda_{t o t}}\right)^{Q_{t h}}+Q_{t h}\left(\frac{\lambda_{d}}{\lambda_{t o t}}\right)^{Q_{t h}+1}\right]}{\lambda_{c}^{2}}
$$

\section{B. Average delay of data packets}

In order to calculate the data packets end-to-end delay, ONUs are modeled using $N$-threshold policy M/G/1 queue [16] with multi-class traffic. For simplicity, we assume that the sleeping time is independent of the arrival process and follows the distribution in (5). This approximation converts our model to $\mathrm{M} / \mathrm{G} / 1$ non-preemptive priority queue with server vacations [17].

The average remaining vacation (sleeping) time can be evaluated as,

$$
\overline{R_{v}}=\frac{\overline{T_{s}^{2}}}{2 \overline{T_{s}}}
$$

while the average remaining service time is,

$$
\bar{R}=\rho_{c} \overline{r_{c}}+\rho_{d} \overline{r_{d}},
$$

where $\overline{r_{c}}=\frac{\overline{X_{c}^{2}}}{2 \overline{X_{c}}}$ and $\overline{r_{d}}=\frac{\overline{X_{d}^{2}}}{2 \overline{X_{d}}}$. We proceed by computing the average waiting time spent in buffer for data packets as follows,

$$
\overline{W_{d}}=\frac{\bar{R}+\left(1-\rho_{c}-\rho_{d}\right) \overline{R_{v}}}{\left(1-\rho_{c}\right)\left(1-\rho_{c}-\rho_{d}\right)}
$$

Finally, the average delay of data packets is,

$$
\overline{D_{d}}=\overline{X_{d}}+\overline{W_{d}}
$$

With the average QoS delay in hand, we can compute $Q_{t h}$ from (8), (11), and (12) numerically.

\section{Simulation Results}

In this section, we show the results obtained from both the analytical model and the simulation. Several parameters were fixed for the simulation part, including the service rate and the simulation time. The data packet is assumed to be

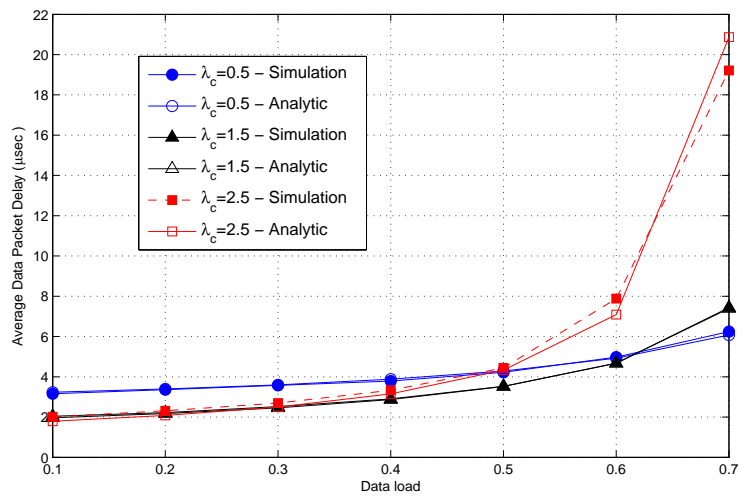

Fig. 2. Average data packet delay vs. data load for $Q_{t h}=20$.

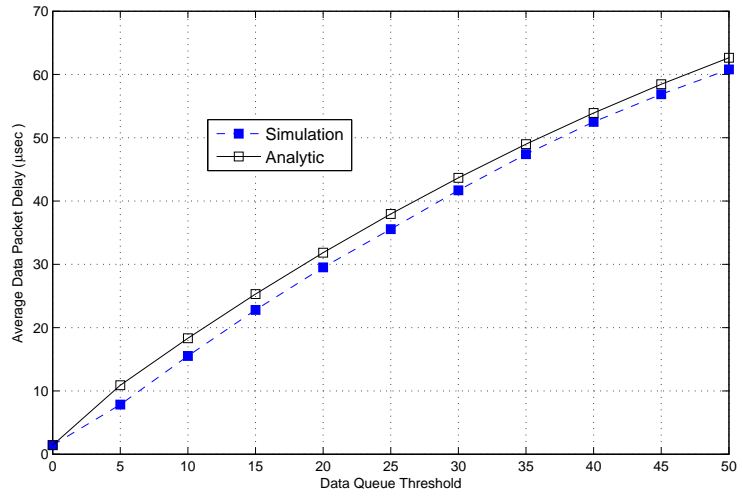

Fig. 3. Average data packet delay vs. $Q_{t h}$ for $\lambda_{c}=0.01 \times 10^{6}$.

exponentially distributed with average size of 1000 bits. The control packet size is assumed to be exponentially distributed with average size of 100 bits. The link rate is set to $1 \mathrm{Gbps}$. Accordingly, the average service time for the data and control packets is $\overline{X_{d}}=1 \mu \mathrm{sec}$ and $\overline{X_{c}}=0.1 \mu \mathrm{sec}$, respectively.

Fig. 2 shows the data packet delay vs. $\rho_{d}$ with different values of $\lambda_{c}$. The threshold for switching from sleeping to active mode (i.e., $\left.Q_{t h}\right)$ is set to 20. For lightly loaded ONU, the delay is more dependent on control packets load rather data packets load. The greater control traffic results in lower data packets delay. This is because the sleeping time depends mostly on control traffic rather than data traffic. As the data load increases, higher control traffic results into higher data packets delay since data packets has lower priority than control packets. The impact of $\rho_{d}$ and $\rho_{c}$ on the data packets delay is shown in Figs. 3 and 4. Fig. 3 shows that data packets delay linearly increase with $Q_{t h}$ at small control traffic. On the other hand, in Fig. 4 the data packets delay tends to saturate with the increase of $Q_{t h}$. The reason for such behavior is that the arrival of control packets triggers the ONU to active mode before reaching $Q_{t h}$. These figures shows how important to estimate the data queue threshold, as underestimation will result in energy waste, while overestimation might violate the QoS 


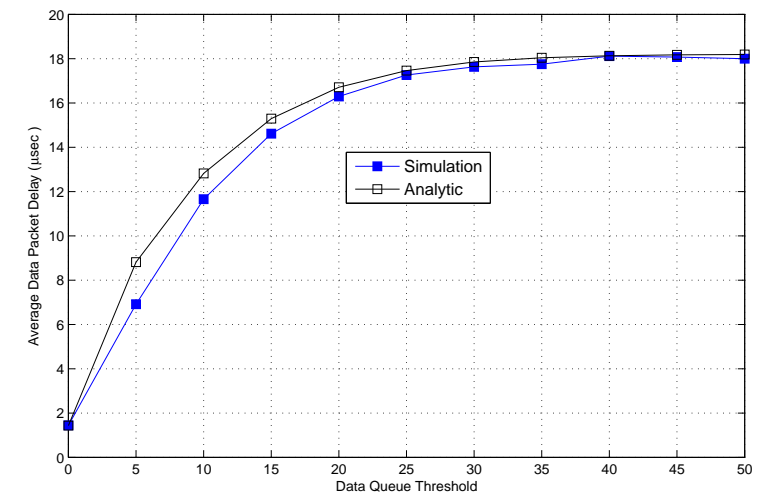

Fig. 4. Average data packet delay vs. $Q_{t h}$ for $\lambda_{c}=0.06 \times 10^{6}$.

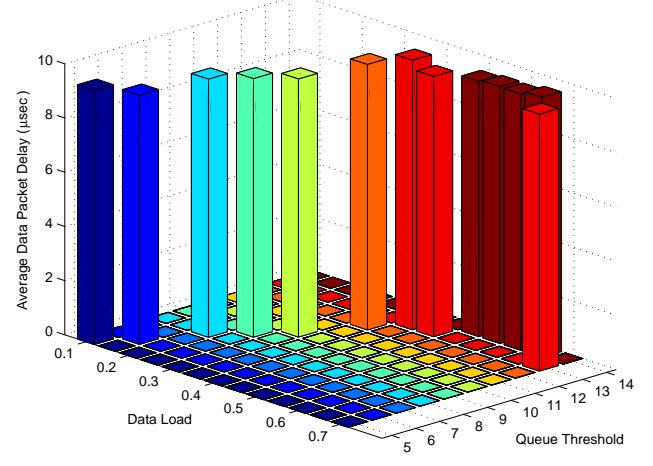

Fig. 5. Average data packet delay under $\mathbb{D}_{Q o S}=10 \mu \mathrm{sec}$

constraints. In order to examine the quality of our scheme, we set $\mathbb{D}_{Q o S}=10 \mu \mathrm{sec}$, loop over different values of data load, and numerically calculate the corresponding $Q_{t h}$ required to maintain the QoS requirement using (8), (11), and (12). Then, we run the simulation with the calculated $Q_{t h}$ to calculate the average data packets delay. Fig. 5 shows that for all values of data load, the average data packets delay never violates the $\mathbb{D}_{Q o S}$ constraint. Instead, our scheme succeeds to dynamically adjust $Q_{t h}$ according to the data load in order to maintain our ultimate objective of maximizing the saved power through the introduced ONU sleeping functionality, while satisfying the imposed average delay constraint.

In Fig. 6 we show the energy saving gain vs. different values of $\mathbb{D}_{Q o S}$ under different loads. We prove that regardless of the QoS delay constraint, our framework achieves equal energy saving gain for some data traffic load. This is because that our framework can successfully decoupled sleeping time from QoS delay when fixing the load.

\section{CONClusion}

In this work, we presented a novel sleep sizing and scheduling framework for energy saving in PON. We showed through both analytical modeling and extensive simulations that our

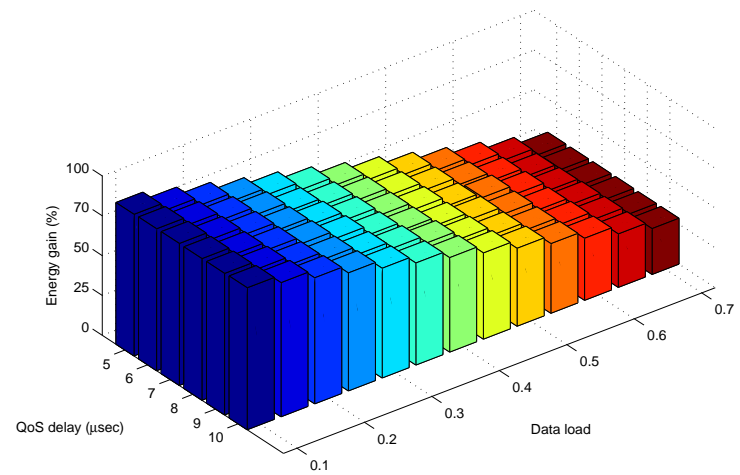

Fig. 6. Energy gain vs. data load for different $\mathbb{D}_{Q o S}$ values

framework can save energy without violating the QoS constraints. To the best of our knowledge, this is the first study that decoupled the sleeping time from QoS delay constraints. This implies that sleeping time portion and consequently the energy saving ratio will only depend on the aggregated traffic load. Our framework can adaptively change the data $Q_{t h}$ to maintain the required $\mathbb{D}_{Q o S}$ without affecting the energy saving gain.

\section{REFERENCES}

[1] Gangxiang Shen, R. S. Tucker, and T. Chae, "Fixed Mobile Convergence (FMC) Architectures for Broadband Access: Integration of EPON and WiMAX," IEEE Communications Magazine, vol. 45 no. 8, pp.44-50, 2007.

[2] Y, Luo, et al., "QoS-aware scheduling over hybrid optical wireless networks," in Proc. of OFC/NFOEC Optical Fiber Communication, pp. 1-7, 2007.

[3] Y. Luo, et al., "Integrating optical and wireless services in the access network," in Proc. of OFC/NFOEC Optical Fiber Communication, 2006.

[4] S. Sarkar, S. Dixit, and B. Mukherjee, "Hybrid Wireless-Optical Broadband-Access Network (WOBAN): A Review of Relevant Challenges," IEEE Journal of Lightwave Technology, vol. 25, no. 11, pp. 3329-3340, 2007.

[5] W. T. Shaw, S. W. Wong, N. Cheng, K. Balasubramanian, X. Zhu, M. Maier, and L. G. Kazovsky, "Hybrid Architecture and Integrated Routing in a Scalable Optical-Wireless Access Network," IEEE Journal of Lightwave Technology, vol. 25, no. 11, pp. 3443-3451, 2007.

[6] G. Kramer, B. Mukherjee, and G. Pesavento, "Ethernet PON (ePON): Design and Analysis of an Optical Access Network", Photonic Network Communications, vol. 3, no. 3, 2001.

[7] Y. Yan, S.-W. Wong, L. Valcarenghi, S.-H. Yen, D. R. Campelo, S. Yamashita, L. Kazovsky, and L. Dittmann, "Energy Management Mechanism for Ethernet Passive Optical Networks (EPONs)," Proc. of IEEE International Conference on Communications, pp. 1-5, 2010.

[8] A. R. Dhaini, "Design and Analysis of Green Mission-Critical FiberWirleless Broadband Access Networks", PhD Thesis, University of Waterloo, ON, Canada, September 2011.

[9] A. R. Dhaini, P.-H. Ho, and G. Shen, "Toward Green Next-Generation Passive Optical Networks," IEEE Communications Magazine, vol. 49, no. 11, pp 94-101, 2011.

[10] A. R. Dhaini, P.-H. Ho, and G. Shen,"Energy Efficiency in Ethernet Passive Optical Networks: For How Long Can ONU Sleep?" University of Waterloo, Tech. Rep., Mar. 2011. [Online]. Available: https://ece. uwaterloo.ca/ adhaini/Files/G_EPON_Sleep.pdf.

[11] J. Zhang and N. Ansari, "Towards energy-efficient 1G-EPON and $10 \mathrm{GEPON}$ with sleep-aware MAC control and scheduling," IEEE Communications, vol. 49, no. 2, 2011.

[12] S. Lee and A. Chen, "Design and analysis of a novel energy efficient ethernet passive optical network," Proc. of International Conference on Networks, pp. 69, 2010. 
[13] R. Kubo, J.-i. Kani, H. Ujikawa, T. Sakamoto, Y. Fujimoto, N. Yoshimoto, and H. Hadama, "Study and demonstration of sleep and adaptive link rate control mechanisms for energy efficient $10 \mathrm{~g}$-epon," IEEE/OSA Journal of Optical Communications and Networking, vol. 2, no. 9, pp. $716729,2010$.

[14] Y. Yan, S.-W. Wong, L. Valcarenghi, S.-H. Yen, D.R. Campelo, S. Yamashita, L. Kazovsky, and L. Dittmann, "Energy management mechanism for ethernet passive optical networks (epons)," Proc. of IEEE International Conference on Communications, pp. 1 5, 2010.

[15] S. Chen, A. Dhaini, P-H. Ho, B. Shihada, G. Shen, C-H. Lin, "Downstream-based Scheduling for Energy Conservation in Green EPONs", Journal of Communications, SI on Future Directions in Computing and Networking, To Appear, 2012.

[16] N. Tian and Z. George Zhang, "Vacation Queueing Models: Theory and Applications, International Series in Operations Research and Management Science. Springer, Dordrecht, 2006.

[17] C.-H. Ng and S. Boon-Hee, "Queueing Modelling Fundamentals: With Applications in Communication Networks, Wiley Publishing, 2nd edition, 2008. 